\title{
AVALIAÇÃO DO DESEMPENHO ISOCINÉTICO DA MUSCULATURA EXTENSORA E FLEXORA DO JOELHO DE ATLETAS DE FUTSAL EM MEMBRO DOMINANTE E NÃO DOMINANTE*
}

\author{
DR. APARECIDO PIMENTEL FERREIRA \\ Professor adjunto do Departamento de Educação Física da Universidade Paulista (Unip) \\ Professor adjunto do Departamento de Educação Física da Universidade Planalto (Uniplan) (Brasília - Brasil) \\ Professor adjunto do Departamento de Enfermagem da Faculdade Integrada Universidade do Instituto \\ Científico de Ensino Superior e Pesquisa (Unlcesp) \\ E-mail: cidopimentel@yahoo.com.br
}

\section{MS. SÉRGIO ADRIANO GOMES}

Mestre em educação física pela Universidade Católica de Brasília (UCB)

Professor adjunto do Departamento de Educação Física do Centro Universitário de Brasília (UniCeub) (Brasilia - Brasil)

E-mail: adrianofutsal@yahoo.com.br

\section{MS. CARLOS ERNESTO SANTOS FERREIRA}

Mestre em educação física pela UCB

Professor adjunto do Departamento de Educação Física da UCB (Brasília - Brasil)

E-mail: carlosf@ucb.br

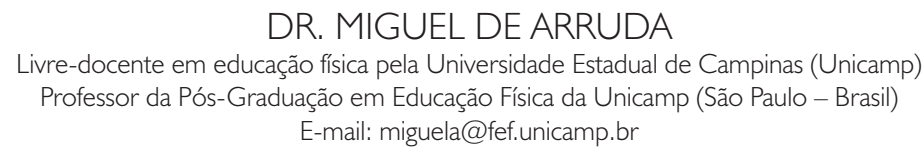

DR. NANCI MARIA DE FRANÇA

Doutora em educação física pela Université Blaise Pascal (França)

Professora da Pós-Graduação em Educação Física da UCB (Brasília - Brasil)

E-mail: nfranca@pos.ucb.br

\section{RESUMO}

Objetivo: avaliar o desempenho isocinético da musculatura extensora e flexora do joelho em atletas de futsal nos membros DO e ND. Metodologia: participaram 23 atletas, com idade de 27,1 $\pm 3,6$ anos, massa corporal 72,7 $\pm 12,6 \mathrm{~kg}$ e estatura I,75 $\pm 6,7 \mathrm{~m}$. Realizou-se 6 repetições máximas nas velocidades de 60, 120, 180 e $300^{\circ} . \mathrm{s}^{-1}$, em ordem crescente no dinamômetro isocinético. Avaliou-se pico de torque (PT), PT normalizado (PTN), PT médio

* O presente trabalho não contou com apoio financeiro de nenhuma natureza e tampouco houve conflitos de interesse para sua realização. 
(PTM), potência média (PM), trabalho total da repetição máxima (TTRM) e quantidade de trabalho total (QTT). Resultados: no movimento de flexão, a PM $180^{\circ} . \mathrm{s}^{-1}$ foi 6,7\% maior no membro DO ( $p<0,05)$. No movimento de extensão, o ND superou o DO $(p<0,05)$, sendo: PT $60^{\circ} \cdot \mathrm{s}^{-1}(+7,6 \%)$, PTN $60^{\circ} \cdot \mathrm{s}^{-1}(+7,0 \%)$, TTRM $120^{\circ} \cdot \mathrm{s}^{-1}(+6,0 \%)$, PM $60^{\circ} \cdot \mathrm{s}^{-1}$ $(+6,6 \%)$ e $120^{\circ} \cdot \mathrm{s}^{-1}(+5,2 \%)$, QTT $60^{\circ} \cdot \mathrm{s}^{-1}(+6,9 \%)$ e $120^{\circ} \cdot \mathrm{s}^{-1}(+7,5 \%)$ e PTM $60^{\circ} \cdot \mathrm{s}^{-1}$ $(+7,3 \%)$ e $120^{\circ} \cdot s^{-1}(+5,2 \%)$. Exceto pela PM e TTRM, todas as variáveis apresentaram maior desempenho a $60^{\circ} \cdot \mathrm{s}^{-1}$. Conclusão: apesar das diferenças entre DO e ND, essas diferenças não os predispõem à incidência de lesões.

PALAVRAS-CHAVE: Atleta; força muscular; potência; desequilíbrio muscular; joelho.

\section{INTRODUÇÃO}

Atualmente, o futsal é o esporte de quadra mais praticado no Brasil (CBFS, 2006), porém poucos estudos envolvendo força, potência e velocidade com essa modalidade foram desenvolvidos (BArbero; Barbero, 2003; Chagas et al., 2005; FERREIRA et al., 2009).

$\bigcirc$ futsal apresenta características de exercícios intermitentes de alta intensidade, seguidos de intervalos incompletos de recuperação, o que resulta em uma maior contribuição das reservas intramusculares de ATP e PCr (BARBERO; BARBERO, 2003). Assim, a força (FONSECA et al., 2007), a potência anaeróbia (COMETTı et al., 200 I) e o índice de fadiga (Ferreira et al., 2009) são variáveis essenciais para atletas de futsal de elite, a fim de manter esforços intensos para atacar e defender.

Alguns autores sugerem que a força, velocidade, potência, resistência, agilidade e massa magra são atributos considerados indispensáveis para o futsal (ALTIMARI et al., I 999, BALIKIAN et al., 2002; ChagAs et al., 2005; GoularT et al., 2007). Nesse sentido, vários testes são utilizados na mensuração de algumas dessas variáveis, incluindo testes de campo, como sprint e squat jump (mais específicos) e testes de laboratórios, como a dinamometria isocinética, que são precisos, porém menos específicos (RIPAMONTI et al., 2008; GREIG et al., 2008). Lehance et al. (2009), analisaram 57 jogadores profissionais e juniores de futebol na pré-temporada, considerando o histórico de lesões, avaliação isocinética e testes de campo, e enfatizam que, embora amplamente utilizada na avaliação da força, a dinamometria isocinética não reflete os aspectos funcionais da prática do futebol, recomendando preferencialmente a utilização de testes funcionais. Todavia, enquanto existe o debate sobre o teste isocinético, envolvendo a eficácia vs funcionalidade em relação à avaliação de desempenho, sua utilidade na avaliação de déficits e desequilíbrios de força muscular, fortemente associado a lesões, não são contestadas. 
Croisier et al. (2008), acompanharam 462 jogadores de futebol profissional (brasileiros, belgas e franceses), avaliados por teste isocinético na pré-temporada, e verificaram que a intervenção originada em detrimento ao desequilíbrio de força constatado na pré-temporada diminuiu a incidência de lesão muscular.

Nesse sentido, o dinamômetro isocinético é um instrumento importante, haja vista que, além da mensuração de variáveis como a força, velocidade, potência e fadiga, adicionalmente, podem apresentar dados importantes que poderão ser utilizados na prevenção das lesões.

O quadríceps e os isquiotibiais são os músculos mais exigidos no futsal, por serem a musculatura mais trabalhada nos tiros curtos, médios e longos, paradas bruscas, chutes, disputas de bolas, saltos, divididas e trancos. Assim, existe a necessidade de se conhecer o desempenho do movimento de flexão e extensão dos joelhos nos membros dominante (DO) e não dominante (ND), pois, constantemente, encontramos jogadores com maior desempenho no membro DO comparado com o ND; e, além da associação com lesões, pode auxiliar no treinamento desportivo e para uma melhor programação dos treinamentos. Dessa forma, o objetivo deste estudo foi avaliar o desempenho isocinético da musculatura extensora e flexora do joelho em atletas da seleção brasiliense de futsal nas velocidades angulares (VAs) 60, 120, 180 e 300 graus por segundo $\left({ }^{\circ} . s^{-1}\right)$ nos membros DO e ND.

\section{MATERIAL E MÉTODOS}

Este estudo é caracterizado como um estudo transversal, do qual participaram uma amostra de 23 atletas convocados para disputar o Campeonato Brasileiro de Seleções, com idade entre 22 e 33 anos, todos integrantes das equipes que disputam - Campeonato Brasiliense. Foram incluídos no estudo todos os atletas convocados pela Federação Brasiliense de Futsal (FBF) que, após testes cardiológicos de repouso e esforço, foram liberados pelo cardiologista.

O estudo foi aprovado pelo Comitê de Ética em Pesquisa da Universidade Católica de Brasília (UCB), 05 I /2005, e constituído por atletas do sexo masculino que assinaram o termo de consentimento livre e esclarecido, e foram considerados aptos a realizar todos os testes de acordo com médico responsável. Os testes foram realizados no Laboratório de Avaliação Física e Treinamento (Lafit).

No laboratório onde os testes foram realizados, a temperatura ambiente foi mantida entre $21^{\circ} \mathrm{C}$ e $23^{\circ} \mathrm{C}$, e a umidade relativa do ar entre $40 \%$ e $60 \%$. Os testes foram realizados nos mesmos horários, entre 9 e I I horas.

Inicialmente, foi realizada a antropometria e posteriormente 0 teste isocinético. A avaliação da composição corporal com as variáveis: massa corporal, 
estatura e dobras cutâneas foram feitas de acordo com os procedimentos descritos por Petroski (1995). Para a estatura e massa corporal, foi utilizada uma balança e estadiômetro marca FilizolaTM Beyond Technology, modelo PL - 200 - (São Paulo, Brasil), com resolução de $0,1 \mathrm{~kg}$ e $0,1 \mathrm{~cm}$. As dobras cutâneas foram mensuradas com um adipômetro Lange Beta Technology Incorpored, Cambridge, Maryland, com resolução de $1 \mathrm{~mm}$. Para a estimativa da densidade corporal, foi utilizada a equação de Jackson e Pollock (1978). A determinação da gordura corporal foi estimada de acordo com a equação de Siri (1956).

\section{TESTE ISOCINÉTICO}

Foi utilizado um dinamômetro isocinético poliarticular, Biodex ${ }^{\circledR}$, modelo System 3 (Biodex, NY, USA), calibrado conforme as especificações e recomendações do fabricante. O mesmo pesquisador conduziu todos os testes, e todos os avaliados realizaram um aquecimento de cinco minutos em uma bicicleta ergométrica marca Lode, modelo Excalibur (Lode - Hol), com uma carga de 60 watts com velocidade de 70 a 80 rpm, seguida de alongamentos, conforme sugerido por Bottaro et al. (2005).

Antes da realização do teste, foi inserida uma sessão de familiarização feita no próprio dinamômetro isocinético com uma série de seis repetições de extensão e flexão de joelho nos membros DO e ND nas VAs 60, 120, 180 e $300^{\circ} \cdot s^{-1}$, aplicadas em ordem crescente. $O$ membro a iniciar o teste DO ou ND foi escolhido por sorteio, e a dominância do membro foi determinada de forma autorreferida.

O teste, com as mesmas VAs da sessão de familiarização, foi realizado com no mínimo 24 e no máximo 72 horas. Nas duas sessões, o encosto do dinamômetro foi ajustado até que a fossa poplítea do joelho estivesse apoiada na parte inferior do assento. ○ eixo de rotação do aparelho foi alinhado com o epicôndilo lateral do fêmur (eixo de rotação anatômico do joelho). $\bigcirc$ encosto da cadeira foi de 90 graus; e o braço de alavanca, ajustado e fixado $2 \mathrm{~cm}$ acima dos maléolos do tornozelo. Os voluntários foram instruídos a não fazerem movimentos de flexão plantar e dorsiflexão, e o individuo foi estabilizado na cadeira com dois cintos no seu tronco, um cinto na pelve e outro na coxa, para evitar contribuição dos membros superiores e a retroversão pélvica, ou mesmo uma possível contribuição de qualquer outra parte do corpo (DVIR, 2002).

A pesagem do membro avaliado relaxado em semiextensão do joelho em 45 graus foi realizada para corrigir a ação da gravidade no movimento de flexão (fator de correção realizado pelo próprio dinamômetro). Uma vez o indivíduo posicionado da forma adequada, foi realizado o movimento de extensão de joelho da perna avaliada em uma taxa de movimento preestabelecida. Todos os avaliados foram igualmente incentivados tanto verbalmente, quanto visualmente pelos avaliadores a desempenhar 
o máximo de força. Ao final do teste, os resultados foram inseridos em uma planilha de dados.

Foi mensurada a fase concêntrica dos músculos extensores e flexores do joelho de ambas as pernas, avaliadas alternadamente pelo dinamômetro isocinético nas VAs 60, 120, 180 e $300^{\circ} \cdot \mathrm{s}^{-1}$. Foi aplicado I minuto de intervalo de recuperação entre as VAs e a escolha da perna (DO ou ND) de início do teste foi feita de forma randômica.

Os parâmetros isocinéticos com seus respectivos valores foram obtidos por um programa computadorizado, que acompanha o equipamento, o que permitiu a determinação do pico de torque (PT), pico de torque normalizado (PTN) - fazendo referência ao pico de torque dividido pela massa corporal, pico de torque médio (PTM) - referente à média de PT das seis repetições, potência média (PM) - referente à média de potência das seis repetições, trabalho total da repetição máxima (TTRM) - referente à repetição mais potente dentre as seis repetições e quantidade de trabalho total (QTT) - referente ao trabalho total de todas as repetições.

\section{ANÁLISE ESTATÍSTICA}

Inicialmente, foi testada a normalidade dos dados por meio do teste de ShapiroWilk. Foi utilizada a estatística descritiva, Teste t pareado e Teste de variância para medidas repetidas, com Least Significant Difference Post Hoc Test (LSD). Em todas as análises, foi adotado o valor de $p<0,05$, para apontar as diferenças significativas. As análises foram realizadas no programa SPSS I I.5 (SPSS Inc., Chicago, IL, USA).

\section{RESULTADOS}

Os dados da caracterização dos 23 atletas da Seleção Brasiliense de Futsal com as variáveis idade, massa corporal, estatura, percentual de gordura corporal (\% GC), bem como seus valores mínimos e máximos, estão apresentados na tabela I.

Tabela I - Caracterização da amostra

\begin{tabular}{|l|c|c|c|}
\hline & Média $\pm \mathrm{DP}$ & Mínimo & Máximo \\
\hline Idade (anos) & $27,1 \pm 3,6$ & 21 & 33,2 \\
\hline Massa corporal $(\mathrm{kg})$ & $72,7 \pm 12,6$ & 57,1 & 112,1 \\
\hline Estatura (cm) & $175,2 \pm 6,7$ & 165 & 188,8 \\
\hline Gordura corporal (\%) & $13,1 \pm 5,6$ & 5,2 & 28,5 \\
\hline
\end{tabular}

Legenda: DP = desvio-padrão.

Os valores médios e seus respectivos desvios-padrão das variáveis PT, PTN, PTM, PM, TTRM e QTT dos membros DO e ND, no movimento de flexão e 
extensão nas VAs 60, 120, 180 e $300^{\circ} \cdot \mathrm{s}^{-1}$, estão apresentados nas tabelas 2 e 3 respectivamente.

Tabela 2 - Valores médios e desvio-padrão das variáveis analisadas pelo teste isocinético no movimento de flexão nas velocidades de $60,120,180$ e $300^{\circ} \cdot s^{-1}$ nos membros DO e ND

\begin{tabular}{|l|c|c|c|c|c|}
\hline & DO/ND & $60^{\circ} \cdot \mathrm{s}^{-1}$ & $120^{\circ} \cdot \mathrm{s}^{-1}$ & $180^{\circ} \cdot \mathrm{s}^{-1}$ & $300^{\circ} \cdot \mathrm{s}^{-1}$ \\
\hline PT $(\mathrm{Nm})$ & $\mathrm{DO}$ & $128,2 \pm 26,3$ & $109,9 \pm 22,6$ & $98 \pm 18,9$ & $78,9 \pm 18$ \\
\hline & $\mathrm{ND}$ & $131 \pm 27,3$ & $108,2 \pm 23,7$ & $96,7 \pm 19,9$ & $76,3 \pm 20,1$ \\
\hline PTN $\left(\mathrm{Nm} \cdot \mathrm{kg}^{-1}\right)$ & $\mathrm{DO}$ & $172,4 \pm 22,1$ & $147,7 \pm 18,2$ & $131,9 \pm 14,8$ & $106,5 \pm 19$ \\
\hline & $\mathrm{ND}$ & $176,4 \pm 22,9$ & $145,3 \pm 19,6$ & $130,3 \pm 18,6$ & $102,4 \pm 20,7$ \\
\hline TTRM (J) & DO & $126,7 \pm 33,8$ & $107,3 \pm 27,9$ & $91,3 \pm 23,5$ & $62,3 \pm 17,9$ \\
\hline & $\mathrm{ND}$ & $126,3 \pm 37,5$ & $107,9 \pm 30,7$ & $89,5 \pm 25,8$ & $60,4 \pm 18,7$ \\
\hline PM(W) & DO & $85 \pm 18,6$ & $129,5 \pm 31,9$ & $147,5 \pm 36,8 *$ & $134,9 \pm 43,4$ \\
\hline & $\mathrm{ND}$ & $86 \pm 17,3$ & $124,6 \pm 32,8$ & $137,6 \pm 38,4$ & $128,1 \pm 43,6$ \\
\hline QTT (J) & DO & $680,4 \pm 202,5$ & $603,3 \pm 160,1$ & $506,1 \pm 129,9$ & $335,6 \pm 102,6$ \\
\hline & $\mathrm{ND}$ & $683,5 \pm 192,3$ & $586,6 \pm 161,3$ & $478,8 \pm 135,8$ & $328,1 \pm 107,3$ \\
\hline PTM (Nm) & DO & $119,5 \pm 26,0$ & $103,1 \pm 21,3$ & $92,3 \pm 17,4$ & $72,7 \pm 17,1$ \\
\hline & ND & $120,9 \pm 23,7$ & $100,5 \pm 22,5$ & $88,7 \pm 18,8$ & $70 \pm 20,9$ \\
\hline
\end{tabular}

Valores em média \pm desvio-padrão.

* Diferença significativa entre o membro DO e ND, $p<0,05$.

Legenda: $\mathrm{DO}=$ membro dominante, $\mathrm{ND}=$ membro não dominante, $\mathrm{PT}=$ pico de torque, $\mathrm{PTN}=$ pico de torque normalizado, $\mathrm{PTM}=$ pico de torque médio, $\mathrm{PM}=$ potência média, $\mathrm{TTRM}=$ trabalho total da repetição máxima, $\mathrm{QTT}=$ quantidade de trabalho total, $\mathrm{Nm}=$ Newton metros, $\mathrm{Nm} \cdot \mathrm{kg}^{-1}=$ Newton metros por quilo, $\mathrm{J}=$ Joules, $\mathrm{W}=$ watts.

Tabela 3 - Valores médios e desvio-padrão das variáveis analisadas pelo teste isocinético no movimento de extensão nas velocidades de 60, 120, 180 e $300^{\circ}$. s- I nos membros DO e N

\begin{tabular}{|c|c|c|c|c|c|}
\hline & $\mathrm{DO} / \mathrm{ND}$ & $60^{\circ} \cdot s^{-1}$ & $120^{\circ} \cdot s^{-1}$ & $180^{\circ} \cdot s^{-1}$ & $300^{\circ} \cdot s^{-1}$ \\
\hline \multirow[t]{2}{*}{$\mathrm{PT}(\mathrm{Nm})$} & DO & $243,4 \pm 46,2 *$ & $199,4 \pm 33,7$ & $170,3 \pm 26$ & $132 \pm 19,3$ \\
\hline & ND & $263,5 \pm 55,6$ & $210 \pm 41,9$ & $175,4 \pm 31,5$ & $134,9 \pm 23,5$ \\
\hline \multirow[t]{2}{*}{ PTN $\left(\mathrm{Nm} \mathrm{kg}^{-1}\right)$} & DO & $329,5 \pm 51,7 * *$ & $270,4 \pm 37,9$ & $231,2 \pm 31,4$ & $179,8 \pm 26,8$ \\
\hline & ND & $354,4 \pm 50,5$ & $282,6 \pm 35,5$ & $236,5 \pm 26,8$ & $|82| \pm 19,9$, \\
\hline \multirow[t]{2}{*}{$\operatorname{TTRM}(\mathrm{J})$} & DO & $179,8 \pm 26,8$ & $209,1 \pm 37,5^{*}$ & $173,8 \pm 29,3$ & $119,3 \pm 20,5$ \\
\hline & ND & $\mid 82,1 \pm 19,9$ & $222,4 \pm 44,6$ & $|82| \pm 36$, & $\mid 23,1 \pm 25,9$ \\
\hline \multirow[t]{2}{*}{$\mathrm{PM}(\mathrm{W})$} & DO & $170,5 \pm 35,3^{*}$ & $249,4 \pm 45,6^{*}$ & $281,8 \pm 54,8$ & $266,6 \pm 57,7$ \\
\hline & ND & $182,6 \pm 39,3$ & $263,1 \pm 53,3$ & $293,6 \pm 63,5$ & $274,5 \pm 69,6$ \\
\hline \multirow[t]{2}{*}{ QTT (J) } & DO & $|343,2 \pm 306,|^{*}$ & $1170,6 \pm 218,4^{*}$ & $970,8 \pm 172,5$ & $632 \pm 158,3$ \\
\hline & ND & $1442,6 \pm 336,3$ & $|265| \pm 258,4$, & $1027,3 \pm 212$ & $689,3 \pm 141$ \\
\hline \multirow[t]{2}{*}{$\mathrm{PTM}(\mathrm{Nm})$} & DO & $230,7 \pm 43,8^{*}$ & $188,2 \pm 30,4^{*}$ & $159,8 \pm 24$ & $124,6 \pm 18,1$ \\
\hline & ND & $248,9 \pm 49,7$ & $198,6 \pm 40,3$ & $\mid 66,1 \pm 31,2$ & $127,7 \pm 24$ \\
\hline
\end{tabular}

Valores em média \pm desvio-padrão.

* Diferença significativa entre o membro DO e ND, $p<0,05$.

Legenda: $\mathrm{DO}=$ membro dominante, $\mathrm{ND}=$ membro não dominante, $\mathrm{PT}=$ pico de torque, $\mathrm{PTN}=$ pico de torque normalizado, $\mathrm{PTM}=$ pico de torque médio, $\mathrm{PM}=$ potência média, TTRM = trabalho total da repetição máxima, QTT= quantidade de trabalho total, $\mathrm{Nm}=$ Newton metros, $\mathrm{Nm}_{\mathrm{kg}}{ }^{-1}=$ Newton metros por quilo, $\mathrm{J}=$ Joules, $\mathrm{W}=$ watts. 
Nas tabelas 2 e 3, é possível verificar que, no movimento de flexão, a PM $180^{\circ}$. $\mathrm{s}^{-1}$ foi 6,7\% maior no membro DO, quando comparado ao ND ( $\left.<<0,05\right)$. Contudo, no movimento de extensão, observou-se que, nas variáveis que apresentaram diferenças significativas, o membro ND superou o DO ( $p<0,05)$, sendo a PT $60^{\circ} \cdot \mathrm{s}^{-1}(7,6 \%)$, PTN $60^{\circ} . s^{-1}(7,0 \%)$, TTRM I $20^{\circ} . s^{-1}(6,0 \%)$, PM $60^{\circ} . s^{-1}(6,6 \%)$ e I $20^{\circ} . s^{-1}(5,2 \%)$, QTT $60^{\circ} . \mathrm{s}^{-1}(6,9 \%)$ e $120^{\circ} . \mathrm{s}^{-1}(7,5 \%)$ e PTM $60^{\circ} . \mathrm{s}^{-1}$ (7,3\%) e $120^{\circ} . \mathrm{s}^{-1}$ (5,2\%).

Os maiores desempenhos foram encontrados na PM no movimento de flexão, a $180^{\circ} \cdot \mathrm{s}^{-1}$, no TTRMa $120^{\circ} \cdot \mathrm{s}^{-1}$ e PMa $180^{\circ} \cdot \mathrm{s}^{-1}$, nos movimentos de extensão, ademais, todas as outras variáveis analisadas (tanto na flexão quanto na extensão) apresentaram maior desempenho na VA $60^{\circ} \cdot \mathrm{s}^{-1}$, perdendo magnitude de acordo com o aumento da VA. A demonstração da cinética de que quanto menor a VA, maior a magnitude do desempenho nas variáveis de PT, PTN, QTT e PTM, diferente da TTRM e PM é mais bem demonstrada na figura I. Os dados apresentados na figura I são referentes à média dos valores de flexão e extensão, ou seja, média da força do quadríceps e isquiotibiais.
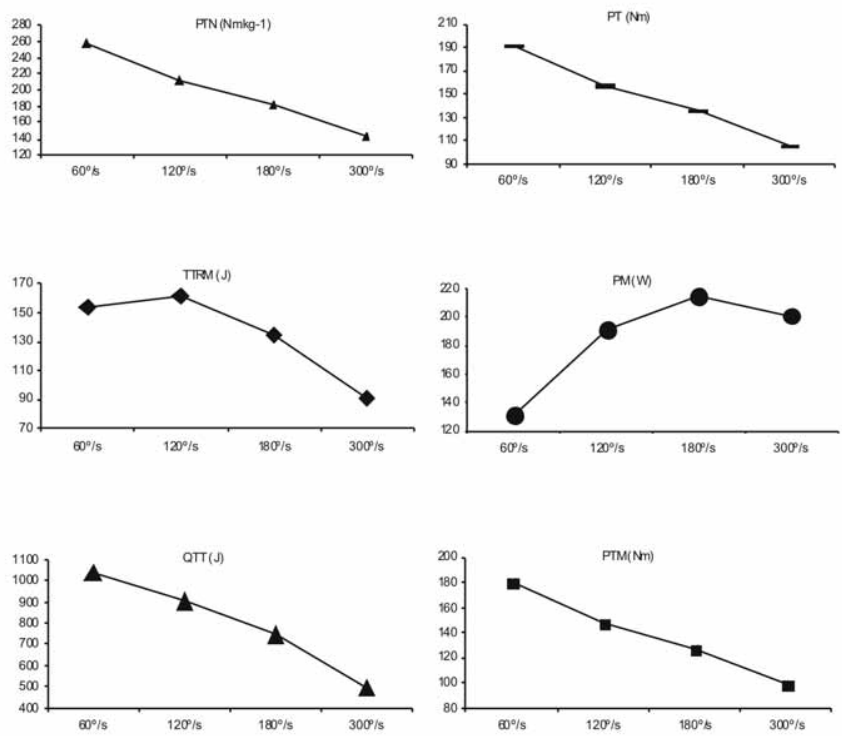

Figura I: Cinética dos valores médios de flexão e extensão do joelho nos membros DO e ND das variáveis do teste isocinético, nas velocidades $60,120,180$ e $300^{\circ} \cdot \mathrm{s}^{-1}$.

Legenda: $\mathrm{DO}=$ membro dominante, $\mathrm{ND}=$ membro não dominante, $\mathrm{PT}=$ pico de torque, PTN = pico de torque normalizado, PTM = pico de torque médio, PM = potência média, TTRM = trabalho total da repetição máxima, QTT $=$ quantidade de trabalho total, $\mathrm{Nm}=$ Newton metros, $\mathrm{Nm} \mathrm{kg}^{-1}=$ Newton metros por quilo, $\mathrm{J}=$ Joules, $\mathrm{W}=$ watts.

Como verificado na figura I, por meio dos valores médios de flexão e extensão (média dos valores do quadríceps e isquiotibiais), analisados conjuntamente nos 
membros DO e ND, nas velocidades 60, 120, I 80 e $300^{\circ} . \mathrm{s}^{-1}$, pode-se perceber que o PT, PTN, QTT e o PTM apresentaram diminuição linear de performance proporcionalmente ao aumento da VA. O TTRM apresentou pequeno aumento na VA $120^{\circ} . \mathrm{s}^{-1}$ em relação a $60^{\circ} . \mathrm{s}^{-1}$ e redução na velocidades de 180 e $300^{\circ} \cdot \mathrm{s}^{-1}$. Já a PM apresentou aumento da magnitude até a VA de $180^{\circ} \cdot \mathrm{s}^{-1}$, com pequena redução a $300^{\circ} \cdot \mathrm{s}^{-1}$.

A diferença entre os membros DO e ND está apresentada na figura 2, a partir da razão DO/ND.

Pode-se observar que, no movimento de extensão em todas as VAs e em todas as variáveis analisadas, o membro ND apresentou desempenho superior ao membro DO (barra branca), apresentando o valor da razão abaixo de 1,0. No movimento de flexão, os dados não apresentam essa mesma lógica, apresentando, inclusive, valores controversos, em que, nas variáveis PT, PTN, PTM e PM, o membro ND apresentou maior desempenho na $V A 60^{\circ} . \mathrm{s}^{-1}$ e menor desempenho em 120, 180 e $300^{\circ} \cdot \mathrm{s}^{-1}$. O TTRM apresentou similaridade de performance nas VAs 60 e $120^{\circ} . \mathrm{s}^{-1}$ e maior desempenho do membro DO nas VAs 180 e $300^{\circ} . \mathrm{s}^{-1}$, enquanto a QTT apresentou similaridade a $60^{\circ} \cdot \mathrm{s}^{-1}$ e maior performance a favor do membro DO nas demais VAs. De maneira mais simplificada, considerando-se todas as variáveis e velocidades angulares, podemos afirmar que, no movimento de extensão, o membro ND foi 100\% mais eficiente do que o membro DO, e, no movimento de flexão, o membro ND foi superior em 33,33\%.
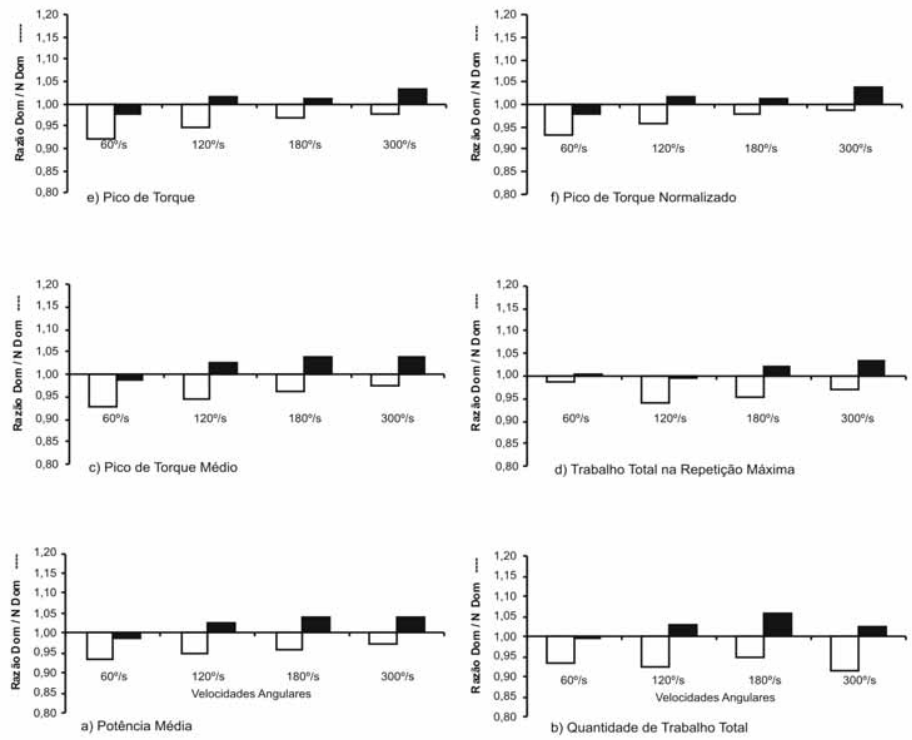

Figura 2: Razão DO/ND das variáveis isocinéticas nas velocidades de $60,120,180$ e $300^{\circ} . s^{-1}$, nos movimentos de flexão e extensão do joelho (barra preta - flexão, barra branca - extensão). 


\section{DISCUSSÃO}

O objetivo deste estudo foi avaliar o desempenho isocinético de atletas da Seleção Brasiliense de Futsal em diversas VAs, nos movimentos de flexão e extensão de joelho dos membros DO e ND. Entretanto, apesar da necessidade de se analisar a força muscular de membros inferiores, devido a sua importância na realização das ações motoras referentes a essa modalidade, nenhum estudo procurou demonstrar essas variáveis em atletas de elite de futsal, dificultando a comparação dos dados do presente estudo com a literatura. Outra dificuldade desse tipo de trabalho é a escassez de dados referentes ao futsal e à mensuração da performance por meio do desempenho isocinético, além da falta de padronização na aplicação dos testes e da variedade de velocidade utilizada.

Comparando-se os valores do presente estudo referente ao PT e PTN com estudos realizados com atletas de futebol brasileiros, verifica-se que os atletas de futsal apresentaram menores valores em ambas as variáveis nas VAs de $60^{\circ} \cdot \mathrm{s}^{-18}$, 180 e $300^{\circ} . s^{-1}$ (FonsECA et al., 2007). Já Lehance et al. (2009), analisando jogadores de futebol belgas, encontraram valores similares e inferiores para profissionais e juniores, respectivamente, no movimento de extensão e similares para o movimento de flexão na $\mathrm{VA} 60^{\circ} \cdot \mathrm{s}^{-1}$.

O presente estudo apresentou uma cinética inversamente proporcional do desempenho comparada às VAs. Esse resultado corrobora com outros, que analisaram diferentes VAs e encontraram comportamento semelhante (GREIG, 2008; SUMIDE et al., 2007). Essa cinética também é verificada em outros grupamentos musculares, como demonstrado por Ripamonti et al. (2008), nas VAs 45, 60, 75, 90, 105 e $120^{\circ} \cdot \mathrm{s}^{-1}$, nos movimentos de flexão e extensão de tronco. Esse fato talvez possa direcionar alguns estudos a testar a magnitude do desempenho isocinético em VAs baixas (ANDERSON et al., 2008).

Contudo, outras VAs também são importantes, pois, de acordo com Hamilton et al. (2008), o PT a $180^{\circ} \cdot \mathrm{s}^{-1}$ teve grande poder de determinação na potência de membros inferiores em alunos/atletas de futebol norte-americanos, explicando 59\% dessa potência. O maior desempenho do PT encontrado nas VAs baixas se deve ao fato de que quanto menor a velocidade angular testada, maior o tempo de contração necessário para se vencer a resistência imposta pelo equipamento; sendo assim, maior a tensão muscular e maior a quantidade de fibras musculares envolvidas, gerando mais força, especialmente no ângulo de maior força.

As variáveis TTRM, QTT e PTM no movimento de extensão, bem como a QTT e PTM no movimento de flexão, demonstrando seus melhores valores na VA $60^{\circ} \cdot \mathrm{s}^{-1}$, quando comparado com as demais VAs, apresentaram a mesma cinética de 
que quanto maior a velocidade, menor o desempenho no dinamômetro isocinético. O TT, tanto na máxima repetição quanto em todas as repetições, representa o produto do torque pelo deslocamento angular, refletindo a energia desenvolvida durante a atividade muscular (SHINZATO et al., 1996).

Em contrapartida, a PM e a TTRM, por sua vez, não apresentaram maior desempenho na menor VA, e a PM, tanto no movimento de extensão quanto no movimento de flexão, apresentou seus maiores valores na VA de $180^{\circ} \cdot \mathrm{s}^{-1}$. Esses dados também são similares a outros estudos que verificaram o maior desempenho de potência em dinamômetro isocinético, ocorrendo em VAs superiores à VA correspondente do maior PT (SUMIDE et al., 2007; RIPAMONTI et al., 2008; HAMILTON et al., 2008). Uma das explicações para esse fenômeno é físico, pois a referida potência corresponde à maior força produzida na menor velocidade e, provavelmente, a velocidade de $180^{\circ} . \mathrm{s}^{-1}$ seja a velocidade ótima para o desenvolvimento dessa variável, pois, em VAs superiores, os atletas de futsal não conseguiriam obter o melhor desempenho de força angular e, em VAs menores, não conseguiriam imprimir velocidade suficiente para manter ótimos valores de potência. Assim, sugere-se que a VA ótima para se treinar a força máxima é a velocidade na qual ocorreu o maior pico de torque $\left(60^{\circ} . \mathrm{s}^{-1}\right)$, e a velocidade ótima para se treinar a potência seja $180^{\circ} \cdot \mathrm{s}^{-1}$. Essa informação é de suma importância no tangente à preparação física e à melhora da performance. Ambas as qualidades físicas são essenciais para a prática do futsal, pois tanto há necessidade de ações máximas como potentes, devido a características da modalidade.

Adicionalmente, além do caráter do desempenho, a avaliação isocinética é importante para se analisar desequilíbrios musculares e possíveis riscos de lesões. Lehance et al. (2009) analisaram 57 jogadores profissionais e juniores de futebol na pré-temporada, considerando o histórico de lesões, avaliação isocinética e testes de campo, e enfatizam que, embora amplamente utilizados na avaliação da força, a dinamometria isocinética não reflete os aspectos funcionais da prática do futebol, recomendando, preferencialmente, a utilização de testes funcionais. No entanto, sugere que a força muscular e a potência anaeróbia podem ser tão importantes para o desempenho como para a prevenção de lesões. Já que sua utilidade na avaliação de déficits e desequilíbrios de força muscular é incontestável, destacando o alto percentual de jogadores que já sofreram lesão, especialmente entre os jovens. Além disso, 64\% dos jogadores já lesionados ainda continuam com desequilíbrio muscular, representando um fator de risco para sofrer outra lesão.

No presente estudo, um dado importante foi a comparação entre DO vs ND (Tabelas 2 e 3 e Figura 2). Podemos verificar que, apesar de algumas variáveis apresentarem diferenças significativas na relação $\mathrm{DO}$ vs $\mathrm{ND}$, tanto no movimento de flexão $(p<0,05)$, quanto de extensão $(p<0,05)$, não são consideradas desequi- 
líbrio muscular, portanto, não sugere risco de lesão, uma vez que o desequilíbrio e o consequente risco de lesão só são considerados quando essa diferença ultrapassa I 5\% (Croisier et al., 2008), ou I0\% (FonsECA et al., 2007; SHINZATO et al., 1996).

Nesse sentido, Croisier et al. (2008), de 2000 a 2005, acompanharam 462 jogadores de futebol profissional brasileiros, belgas e franceses, objetivando determinar se as variáveis da força avaliadas por teste isocinético na pré-temporada poderia ser preditoras da subsequente lesão muscular, e se a normalização dos desequilíbrios de força, também verificados na pré-temporada, poderia reduzir a incidência de lesões. A taxa de lesão muscular foi significativamente maior nos indivíduos que apresentaram desequilíbrios de força não tratada, em comparação com os jogadores sem nenhum desequilíbrio verificado na pré-temporada, apresentando risco relativo de 4,7 vezes mais chance de lesão. $\bigcirc$ risco de lesão permaneceu significativamente alto nos jogadores com desequilíbrio e que participaram de um trabalho de compensação, porém sem um controle posterior (risco relativo de 2,9 vezes mais chance de lesão). Em contrapartida, a normalização dos parâmetros isocinéticos reduziu o risco de lesão, quando comparado a jogadores sem desequilíbrios (risco relativo I,4).

Essa diferença de desempenho encontrada no presente estudo entre DO vs ND poderia explicar, por exemplo, a diferença encontrada na performance do chute de membros dominante e não dominante, descritas por Barbieri et al. (2008), e ser utilizada para a prescrição de treinamento de força tanto para a região do quadríceps, quanto para os isquiotibiais, contudo, não ultrapassa os pontos de corte estabelecidos (CroisIER et al. 2008; FonSECA et al., 2007; SHINZATO et al., 1996) para oferecer risco de lesão, mostrando que os atletas brasilienses estão com um bom equilíbrio muscular.

Uma sugestão para próximos trabalhos nessa linha é a execução de pesquisas longitudinais, que possam verificar os efeitos dos treinamentos realizados nas VAs correspondentes ao maior pico de torque e maior potência, e em VA intermediária, a fim de verificar qual velocidade de treinamento pode proporcionar melhoras nas duas variáveis (PT e PM), haja vista que ambas são extremamente importantes para a avaliação da performance no futsal de elite; além disso, dados referentes ao desequilíbrio de força entre jogadores de futsal, determinados em pré-temporada, com acompanhamento por uma ou duas temporadas, devem ser realizados a fim de traçar um perfil específico do futsal relacionado ao risco de aparecimento de lesões.

\section{CONCLUSÃO}

Exceto a PM e a TTRM, todas as variáveis analisadas apresentaram maior desempenho a $60^{\circ} \cdot \mathrm{s}^{-1}$, tanto na flexão como na extensão. Na flexão, a PM foi maior no membro dominante, na extensão as variáveis de PT, PTN, TTRM, PM, QTT 
e PTM foram maiores no membro não dominante. Contudo, apesar de essas variáveis apresentarem diferenças significativas entre os membros, essas diferenças foram abaixo de 10\%, o que, por essa análise, não os predispõem a lesões.

\section{Evaluate of dominant and nondominant knee extensors and flexors isokinetic performance in futsal athletes}

ABSTRACT: Objective: to evaluate the performance of isokinetic muscle extensor and flexor of the knee in athletes of futsal in DO and ND members. Methods: 23 athletes, with age $27.1 \pm 3.6$ years, body mass $72.7 \pm 12.6 \mathrm{~kg}$ and height $1.75 \pm 6.7 \mathrm{~m}$ participated of study. Were performed 6 maximum repetitions at 60, 120, 180 e $300^{\circ} . \mathrm{s}^{-1}$ speeds, in ascending order on the isokinetic dynamometer. We evaluated peak torque (PT), normalized PT (PTN), medium PT (PTM), mean power (MP), total work of repetition maximum (TTRM) and total work (QTT). Results: in flexion movement, the PM $180^{\circ} . \mathrm{s}^{-1}$ was $6.7 \%$ higher in the DO member $(p<0.05)$. In extension the ND was higher than the DO $(p<$ 0.05), as follows: PT $60^{\circ} \cdot \mathrm{s}^{-1}(+7,6 \%)$, PTN $60^{\circ} \cdot \mathrm{s}^{-1}(+7,0 \%), \operatorname{TTRM} 120^{\circ} \cdot \mathrm{s}^{-1}(+6,0 \%)$, PM 60.$s^{-1}(+6,6 \%)$ e $120^{\circ} \cdot s^{-1}(+5,2 \%)$, QTT $60^{\circ} \cdot s^{-1}(+6,9 \%)$ e $120^{\circ} \cdot s^{-1}(+7,5 \%)$ e PTM $60^{\circ} \cdot \mathrm{s}^{-1}(+7,3 \%)$ e $120^{\circ} \cdot \mathrm{s}^{-1}(+5,2 \%)$. Except for PM and TTRM all variables showed higher performance to $60^{\circ} \cdot \mathrm{s}^{-1}$. Conclusion: despite the differences between DO vs ND, these differences did not predispose the incidence of injuries.

KEYWORDS: Athlete; muscular strength; power; muscular imbalance; knee.

La evaluación del desempeño isocinético de la musculatura extensora y flexora de la rodilla em jugadores de fútbol sala en miembro dominante y no dominante

RESUMEN: Objetivo: evaluar el desempeño isocinético de los musculaos extensores y flexores de la rodilla en atletas de fútbol sala en miembro dominante y no dominante. Métodos: 23 atletas, con edad 27, I \pm 3,6 años, la masa corporal 72,7 $\pm 12,6 \mathrm{~kg}$ y la altura de 1,75 $\pm 6,7 \mathrm{~m}$ participaron del estudio. Se realizó 6 repeticiones máximas, a velocidades de 60 , 120, 180 y $300^{\circ} . \mathrm{s}^{-1}$, en orden ascendente en el dinamómetro isocinético. Se evaluó: pico de torque (PT), PT normalizado (PTN), PT medio (PTM), potencia media (PM), trabajo total de la repetición máxima (TTRM) y cantidad de trabajo total (CTT). Resultados: el movimiento de flexión, la PM $180^{\circ} . s^{-1}$ fue de 6,7\% mayor en el miembro DO $(p<0,05)$. En el movimiento de extensión el miembro ND superó el de DO $(p<0,05)$, de la siguiente manera: PT $60^{\circ} \cdot \mathrm{s}^{-1}(+7,6 \%)$, PTN $60^{\circ} \cdot \mathrm{s}^{-1}(+7,0 \%)$, TTRM $120^{\circ} \cdot \mathrm{s}^{-1}(+6,0 \%)$, PM $60^{\circ}$. $\mathrm{s}^{-1}(+6,6 \%)$ e $120^{\circ} \cdot \mathrm{s}^{-1}(+5,2 \%)$, QTT $60^{\circ} \cdot \mathrm{s}^{-1}(+6,9 \%)$ e $120^{\circ} \cdot \mathrm{s}^{-1}(+7,5 \%)$ e PTM $60^{\circ} \cdot \mathrm{s}^{-1}(+7,3 \%)$ e $120^{\circ} \cdot \mathrm{s}^{-1}(+5,2 \%)$. Excepto por la PM y TTRM todas las variables presentaron mayor desempeño a $60^{\circ} \cdot \mathrm{s}^{-1}$. Conclusión: a pesar de las diferencias entre DO y ND, estas diferencias no predisponen a la incidencia de las lesiones.

PALABRAS CLAVES: Atleta; fuerza muscular; potencia; desequilibrio muscular; rodilla. 


\section{REFERÊNCIAS}

ALTIMARI, L. R.; OKANO, A. C.; COELHO, D.F.; CYRINO, E. S. Efeitos do treinamento de futsal sobre o desempenho motor em atividades predominantemente aeróbias e anaeróbias. Revista Treinamento Desportivo, v. 4, n. 3, p. 23-28, 1999.

ANDERSSON, H.; RAASTAD, T.; NILSSON, J.; PULSEN, G.; GARTHE, I.; KADI, F. Neuromuscular fatigue and recovery in elite female soccer: effects of active recovery. Medicine and Science in Sports and Exercise, v. 40, n. 2, p. 372-80, 2008.

BALIKIAN, P.; LOURENÇÃO, A.; RIBEIRO, L. F. P.; FESTUCCIA, W. T. L.; NEIVA, C. M. Consumo máximo de oxigênio e limiar anaeróbio de jogadores de futebol: comparação entre as diferentes posições. Revista Brasileira de Medicina do Esporte, v. 8, n. 2, p. 32-36, 2002.

BARBERO ÁLVAREZ, J. C.; BARBERO ÁLVAREZ, V. Relación entre el consumo máximo de oxígeno y la capacidad para realizar ejercicio intermitente de alta intensidad en jugadores de fútbol sala. Revista de Entrenamiento Deportivo, v. 17, n. 2, p. 13-24, 2003.

BARBIERI, F. A.; SANTIAGO, P. R. P.; GOBBI, L. T. B.; CUNHA, A. S. Diferenças entre o chute realizado com o membro dominante e não dominante no futsal: variabilidade, velocidade linear das articulações, velocidade da bola e desempenho. Revista Brasileira de Ciências do Esporte, v. 29, n. 2, p. 129-146, 2008.

BOTTARO, M.; RUSSO, A. F.; OLIVEIRA, R. J. The effects of rest interval on quadriceps torque during an isokinetic testing protocol in elderly. Journal of Sports Science and Medicine, v. 4, p. 285-290, 2005.

CHAGAS, M. H.; LEITE, C. M. F.; UGRINOWITSCH, H.; BENDA, R. N.; MENZEL, H.-J.; SOUZA, P. R. C.; MOREIRA, E. A. Associação entre tempo de reação e de movimento em jogadores de futsal. Revista Brasileira de Educação Física e Esporte, v. 19, n. 4, p. 269-75, 2005.

COMETTI, G.; MAFFIULETTI, N. A.; POUSSON, M.; CHATARD, J. C.; MAFFULLI, N. Isokinetic strength and anaerobic power of elite, subelite and amateur French soccer players. International Journal of Sports Medicine, v. 22, p. 45-51, 2001.

CONFEDERAÇÃO BRASILEIRA DE FUTEBOL DE SALÃO - CBFS. Site oficial. Disponível em: <www.cbfs.com.br>. Acesso em: 14 jun. 2006.

CROISIER, J.; GANTEAUME, S.; BINET, J.; GENTY, M.; FERRET, J. Strength imbalances and prevention of hamstring injury in professional soccer players: a prospective study. American Journal Sports Medicine, v. 36, n. 8, p. 1.4691.475, 2008.

DVIR, Z. Isocinética: avaliações musculares, interpretações e aplicações clínicas. São Paulo: Manole, 2002. 
FERREIRA, A. P.; GOMES, A. S.; LANDHWER, R.; FRANÇA, N. M. Potência anaeróbia e índice de fadiga de atletas de futsal da Seleção Brasiliense. Revista Brasileira de Futebol, v. 2, n. I, p. 60-69, 2009.

FONSECA, S. T.; OCARINO, J. M.; SILVA, P. L. P.; BRICIO, R. S.; COSTA, C. A.; WANNER, L. L. Caracterização da performance muscular em atletas profissionais de futebol. Revista Brasileira de Medicina do Esporte, v. 13, n. 3, p. |43-147, 2007.

GOULART, L. F; DIAS, R. M. R.; ALTIMARI, L. R. Força isocinética de jogadores de futebol categoria sub-20: comparação entre diferentes posições de jogo. Revista Brasileira de Cineantropometria e Desempenho Humano, v. 9, n. 2, p. 165-169, 2007.

GREIG, M. The influence of soccer-specific fatigue on peak isokinetic torque production of the knee Flexors and extensors. American Journal of Sports Medicine, v. 36, n. 7, p. 1.403I.409, 2008.

HAMILTON, R. T.; SHULTZ, S. J.; SCHMITZ, R. J.; PERRIN, D. H. Triple-hop distance as a valid predictor of lower limb strength and power. Journal of Athletic Training, v. 43, n. 2, p. |44-|5|, 2008.

JACKSON, A. S.; POLLOCK, M. L. Generalized equations for predicting body density of men. British Journal of Nutrition, v. 40, n. 3, p. 497-504, 1978.

LEHANCE, C.; BINET, J.; BURY, T.; CROISIER, J. Muscular strength, functional performances and injury risk in professional and junior elite soccer players. Scandinavan Journal of Medicine and Science in Sports, v. 19, n. 2, p. 243-251, 2009.

PETROSKI, E. Desenvolvimento e validação de equações generalizadas para estimativa da densidade corporal em adultos. Tese (Doutorado) - Centro de Educação Física e Desportos, Universidade Federal de Santa Maria, Santa Maria, 1995.

RIPAMONTI, M.; COLIN, D.; RAHMANI, A. Torque-velocity and power-velocity relationships during isokinetic trunk flexion and extension. Clinical biomechanics (Bristol, Avon), v. 23, n. 5, p. 520-526, 2008.

SHINZATO, G.T.; VASCONCELOS, J. C. P.; OGAWA, C. T.; SAMPAIO, I. C. S. P.; GONÇALVES, A.; NEVES, E. M. Protocolo de avaliação funcional de joelho em patologias ortopédicas. Acta Fisiatrica, v. 3, n. I, p. 30-36, 1996.

SIRI, W. E. Body composition from fluid spaces and density: analysis of methods - 196I. Nutrition, v. 9, n. 5, p. 480-491, 1965.

SUMIDE, T.; SAKURABA, K.; SAWAKI, K.; OHMURA, H.; TAMURA, Y. Effect of resistance exercise training combined with relatively low vascular occlusion. Journal of Science and $\mathrm{Me-}$ dicine in Sport, v. 12, n. I, p. 107-1 12, 2007. 
Recebido: 29 jul. 2009

Aprovado: 3 mar. 2010

Endereço para correspondência:

Aparecido Pimentel Ferreira

SCLN, 106, Bloco A, 54, ap. 212

Brasília-DF

CEP 70742-5 I0 